Article

\title{
Nuclear Space Facts, Strange and Plain
}

\author{
Jeremy Becnel ${ }^{1, *, \dagger}$ and Ambar Sengupta ${ }^{2, \dagger}$ \\ 1 Department of Mathematics, Stephen F. Austin State University, PO Box 13040 SFA Station, \\ Nacogdoches, TX 75962, USA \\ 2 Department of Mathematics, University of Connecticut, 341 Mansfield Road U1009, Storrs, \\ CN 06269-1009, USA; sengupta@math.lsu.edu \\ * Correspondence: becneljj@sfasu.edu; Tel.: +1-936-468-1582; Fax: +1-936-468-1669 \\ + These authors contributed equally to this work.
}

Academic Editor: Palle E.T. Jorgensen

Received: 31 May 2016; Accepted: 27 September 2016; Published: 9 October 2016

\begin{abstract}
We present a scenic but practical guide through nuclear spaces and their dual spaces, examining useful, unexpected, and often unfamiliar results both for nuclear spaces and their strong and weak duals.
\end{abstract}

Keywords: nuclear spaces; topological vector spaces; dual spaces

\section{Introduction}

The purpose of this paper is to present an exploration of features of the type of nuclear space structures that arise in applications. Nuclear spaces provide a convenient setting for infinite dimensional analysis and have been used in mathematical quantum field theory (Glimm and Jaffe [1] and Rivasseau [2], for instance) and in stochastic analysis (see, for instance, Itô [3] or Chiang et al. [4]). These applications involve probability measures defined on duals of nuclear spaces.

The theory of nuclear spaces was developed by Grothendieck [5] and since then most traditional works [6-10] on nuclear spaces have stressed the interplay of the algebraic structure and the topological structure, such as, for example, in defining and studying tensor products. Our focus is quite different, the motivation coming from topological questions that are relevant to the study of measures on duals of nuclear spaces. Firmly avoiding the study of nuclear spaces in generality or explore results in the greatest generality, we get down to concrete results and questions very quickly. Indeed, our focus is not on "bare" nuclear spaces in themselves but such spaces with additional structure, specifically a chain of Hilbert spaces that are present in the context of applications in which we are interested.

We take an unorthodox approach to the presentation of results, stating, and justifying as needed, facts, some of which are negative answers to questions that arise naturally when working with nuclear spaces. Most facts are stated in terms of the nuclear space or its dual. However, on occasion, results are easily developed in a broader setting and are thus presented in more generality. We also present some unknown results and examples (some of the negative variety) to illustrate important properties of nuclear spaces and their duals.

\section{Topological Vector Spaces}

We begin with a summary of some essential notions and facts about topological vector spaces. We refer to [11] for proofs.

By a topological vector space we mean a real or complex vector space $X$, equipped with a Hausdorff topology for which the operations of addition and multiplication are continuous.

A set $C$ in a vector space (topological vector space) is said to be convex if given any $x, y \in C$, the linear combination $t x+(1-t) y \in C$ for all $t \in[0,1]$. A locally convex space is a topological 
vector space in which every neighborhood of 0 contains a convex neighborhood of 0 . A convex neighborhood $V$ of 0 is balanced if it closed under multiplication by scalars of magnitude $\leq 1$. Every convex neighborhood of 0 contains a balanced convex neighborhood of 0 , and it is the latter type of neighborhood that is most useful.

\subsection{Seminorms from Neighborhoods}

Let $V$ is a balanced convex neighborhood of 0 and define $\rho_{V}$ by way of

$$
\rho_{V}(x)=\inf \{t>0: x \in t V\} .
$$

Since $V$ is a neighborhood of 0 there is a small enough positive scaling of $x$ that makes it fall within $V$, and so there is a large enough scaling of $V$ that includes $x$; thus $\rho_{V}(x)<\infty$. Since $V$ is balanced and convex it follows that $\rho_{V}$ is a semi-norm:

$$
\begin{aligned}
\rho_{V}(x+y) & \leq \rho_{V}(x)+\rho_{V}(y) \\
\rho_{V}(\lambda x) & =|\lambda| \rho_{V}(x)
\end{aligned}
$$

for all $x, y \in X$ and scalar $\lambda$.

A set $D \subset X$ is said to be bounded if $D$ lies inside a suitably scaled up version of any given neighborhood of 0 ; thus, $D$ is bounded if for any neighborhood $V$ of 0 there is a $t>0$ such that $D \subset t V$. If $X$ is locally convex then $D \subset X$ is bounded if and only if $\rho_{U}(D)$ is a bounded subset of $[0, \infty)$ for every convex balanced neighborhood $U$ of 0 .

As usual, a set $K$ in the topological vector space $X$ is said to compact if every open cover of $K$ has a finite subcover. A sequence $\left(x_{n}\right)$ in $X$ is said to be Cauchy if the differences $x_{n}-x_{m}$ eventually lie in any given neighborhood of 0 , and $X$ is said to be complete if every Cauchy sequence converges. Also, $X$ is said to be separable if there exists a countable set $Q \subset X$ such that every nonempty open subset of $X$ contains at least one element of $D$.

If the topology on $X$ is metrizable then there is a metric that induces the topology, is translation-invariant, and for which open balls are convex. If, moreover, $X$ is also complete then such a metric can be chosen for which every metric-Cauchy sequence is convergent.

\subsection{Dual Spaces}

The dual space $X^{\prime}$ of a topological vector space $X$ is the vector space of all continuous linear functionals on $X$ (these functionals take values in the field of scalars, $\mathbb{R}$ or $\mathbb{C}$ ).

If $X$ is locally convex then the Hahn-Banach theorem guarantees that $X^{\prime} \neq\{0\}$ if $X$ itself is not zero.

There are several topologies of interest on $X^{\prime}$. For now let us note the two extreme ones:

- Using $\mathbb{F}$ to denote the field of scalars, the weak topology on $X^{\prime}$ is the smallest topology on $X^{\prime}$ for which the evaluation map

$$
X^{\prime} \rightarrow \mathbb{F}: x^{\prime} \mapsto\left\langle x^{\prime}, x\right\rangle
$$

is continuous for all $x \in X$. This topology consists of all unions of translates of sets of the form

$$
B(D ; \epsilon)=\left\{x^{\prime} \in X^{\prime}: \sup _{x \in D}\left|\left\langle x^{\prime}, x\right\rangle\right|<\epsilon\right\}
$$

with $D$ running over finite subsets of $X$, and $\epsilon$ over $(0, \infty)$.

- The strong topology on $X^{\prime}$ consists of all unions of translates of sets of the form

$$
B(D ; \epsilon)=\left\{x^{\prime} \in X^{\prime}: \sup _{x \in D}\left|\left\langle x^{\prime}, x\right\rangle\right|<\epsilon\right\}
$$

with $D$ running over all bounded subsets of $X$ and $\epsilon$ over $(0, \infty)$. 
The remainder of this section provides context for the rest of the paper but is not actually used later.

\subsection{An Associated Chain of Banach Spaces}

It is clear that the set $\rho_{V}^{-1}(0)$ of vectors of semi-norm zero form a vector subspace of $X$. The quotient space

$$
X / \rho_{V}^{-1}(0)
$$

is a normed linear space, with norm $\|\cdot\|_{V}$ induced from $\rho_{V}$ :

$$
\|a\|_{V}=\rho_{V}(\tilde{a})
$$

where $\tilde{a}$ denotes any element in $X$ that projects down to $a$; the value $\|a\|_{V}$ is independent of the specific choice of $\tilde{a}$. We denote by $X_{V}$ the Banach space obtained by completion of this space:

$$
X_{V}=\overline{X / \rho_{V}^{-1}(0)}
$$

and let $p_{V}$ denote the quotient projection, viewed as a map of $X$ into $X_{V}$ :

$$
p_{V}: X \rightarrow X_{V}: x \mapsto x+\rho_{V}^{-1}(0)
$$

In some cases of interest $\rho_{V}$ itself is a norm, in which case $p_{V}$ is actually an injection into the completion of $X$ relative to this norm.

Thus for any locally convex topological vector space $X$ there is associated a system of Banach spaces $X_{U}$, these arising from convex balanced neighborhoods $U$ of 0 in $X$.

If $U$ and $V$ are convex, balanced neighborhoods of 0 in $X$, and $U \subset V$, then

$$
\left\|p_{V}(x)\right\|_{V}=\rho_{V}(x) \leq \rho_{U}(x)=\left\|p_{U}(x)\right\|_{U,}
$$

and so there is a well-defined contractive linear mapping

$$
p_{V U}: X_{U} \rightarrow X_{V}
$$

specified uniquely by requiring that it maps $p_{U}(x)$ to $p_{V}(x)$ for all $x \in X$.

A complete, metrizable, locally convex space $X$ is obtainable as a 'projective limit' of the Banach spaces $X_{U}$ and the system of maps $p_{V U}$. We will not need a general understanding of a projective limit; for our purposes let us note that if for each convex balanced neighborhood $U$ of 0 in $X$ an element $x_{U} \in X$ is given, such that $p_{V U}\left(x_{U}\right)=x_{V}$ whenever $U \subset V$, then there is an element $x \in X$ such that $x_{U}=p_{U}(x)$ for all $x \in X$.

With $X$ as above, let us choose a sequence of neighborhoods $U_{n}$ of 0 , with each $U_{n}$ balanced and convex, such that every neighborhood of 0 contains some $U_{k}$ and

$$
U_{1} \supset U_{2} \supset \cdots
$$

By (6) we have the chain of spaces

$$
\ldots X_{U_{3}} \rightarrow X_{U_{2}} \rightarrow X_{U_{1}}
$$

where the mappings are of the form $p_{U_{n} U_{n+1}}$. Thus we can view $X$ as obtained from this sequence of spaces.

Complete metrizable nuclear spaces arise in the case where the spaces $X_{U_{n}}$ are Hilbert spaces and the mappings $X_{U_{n+1}} \rightarrow X_{U_{n}}$ are all Hilbert-Schmidt, mapping the closed unit ball in $X_{U_{n+1}}$ into an ellipsoid in $X_{U_{n}}$ whose semiaxes lengths form a square-summable sequence. 


\section{The Nuclear Space Structure}

We work with a real or complex infinite dimensional topological vector space $\mathcal{H}$ equipped with additional structure as we now describe. We assume that there is a sequence of inner-products $\langle\cdot, \cdot\rangle_{p}$ for $p \in\{0,1,2,3, \ldots\}$, on $\mathcal{H}$ such that

$$
\|\cdot\|_{0} \leq\|\cdot\|_{1} \leq \cdots
$$

The completion of $\mathcal{H}$ in the norm $\|\cdot\|_{0}$ is denoted $H_{0}$, and inside this Hilbert space the completion of $\mathcal{H}$ with respect to $\|\cdot\|_{p}$ is a dense subspace of $H_{0}$ denoted by $H_{p}$. We assume that $H_{0}$ is separable, and that $\mathcal{H}$ is the intersection of all the spaces $H_{p}$. Thus,

$$
\mathcal{H}=\bigcap_{p=0}^{\infty} H_{p} \subset \cdots \subset H_{2} \subset H_{1} \subset H_{0} .
$$

(This is a special form of the chain of spaces seen in (7).) Furthermore, we assume that each inclusion $H_{p+1} \rightarrow H_{p}$ is a Hilbert-Schmidt operator (such operators are discussed below in Section 3.1). Then there is an orthonormal basis $v_{1}, v_{2}, \ldots$ in $H_{p+1}$ for which

$$
\sum_{n=1}^{\infty}\left\|v_{n}\right\|_{p}^{2}<\infty
$$

We denote by $\tau_{p}$ is the topology on $\mathcal{H}$ given by $\|\cdot\|_{p}$. By (8), the identity map

$$
\left(\mathcal{H}, \tau_{p+1}\right) \rightarrow\left(\mathcal{H}, \tau_{p}\right)
$$

is continuous, for $p \in\{0,1,2, \ldots\}$, and so

$$
\tau_{0} \subset \tau_{1} \subset \cdots .
$$

The inclusions here are strict because of the Hilbert-Schmidt assumption made above (bearing in mind that $\mathcal{H}$ is infinite-dimensional).

Because of the relations (11), the union $\tau$ of all the topologies $\tau_{p}$ is also a topology. Thus a subset of $\mathcal{H}$ is open if and only if it is the union of open $\|\cdot\|_{p}$-balls with $p$ running over some subset of $\{0,1,2, \ldots\}$. This topology makes $\mathcal{H}$ a topological vector space. Moreover, $\mathcal{H}$, with this topological vector space structure, is the projective limit from the inclusions $\mathcal{H} \rightarrow H_{p}$. We shall not use any general facts or theories of projective limits.

We take $\mathcal{H}$ as the nuclear space we work with; thus in our discussions the nuclear space comes equipped with the additional structure of the Hilbert norms $\|\cdot\|_{p}$.

An infinite-dimensional topological vector space is never locally compact. That is, points do have necessarily have compact neighborhoods. However, a nuclear space is an excellent substitute in the infinite-dimensional case because it satisfies the Heine-Borel property as we shall see in Fact 14.

\subsection{Hilbert-Schmidt Operators}

We will use Hilbert-Schmidt operators, and recall here some standard facts about them. For a linear operator $T: H \rightarrow K$ between Hilbert spaces, define the Hilbert-Schmidt norm to be

$$
\|T\|_{\mathrm{HS}} \stackrel{\text { def }}{=}\left\{\sum_{v \in \mathcal{B}}\|T v\|^{2}\right\}^{1 / 2}<\infty
$$

where $\mathcal{B}$ is some orthonormal basis of $H$; if $H=0$ the Hilbert-Schmidt norm of $T$ is, by definition, 0 . If $\|T\|_{\mathrm{HS}}<\infty$ then $T$ is said to be a Hilbert-Schmidt operator. The following facts are well known (see, for instance, [12-15]): 
Fact 1. For a linear operator $T: H \rightarrow K$ between Hilbert spaces, the Hilbert-Schmidt norm given in (12) is independent of the choice of the orthonormal basis $\mathcal{B}$. If $T$ is Hilbert-Schmidt, then:

(i) $T$ is bounded and $\|T\| \leq\|T\|_{\mathrm{HS}}$;

(ii) the adjoint $T^{*}: K \rightarrow H$ is also Hilbert-Schmidt and $\left\|T^{*}\right\|_{\mathrm{HS}}=\|T\|_{\mathrm{HS}}$;

(iii) the image of any closed ball in $H$ under $T$ is a compact subset of $K$;

(iv) if $T$ is injective then $H$ is separable;

(v) if $H \neq 0$ then there is an orthonormal basis of $H$ consisting of eigenvectors of $T^{*} T$ (see [15] ( $\left.p .28\right)$ ).

In the context of $(\mathrm{v})$ consider an orthonormal sequences $e_{1}, e_{2}, \ldots$ in $H$ consisting of eigenvectors of $T^{*} T$ :

$$
T^{*} T e_{n}=\lambda_{n} e_{n}
$$

for all $n \geq 1$, where $\lambda_{n}$ are eigenvalues of $T^{*} T$; then

$$
\left\langle T e_{n}, T e_{n}\right\rangle_{H}=\left\langle e_{n}, T^{*} T e_{n}\right\rangle_{H}=\lambda_{n}\left\langle e_{n}, e_{n}\right\rangle_{H}=\lambda_{n}
$$

and, similarly,

$$
\left\langle T e_{n}, T e_{m}\right\rangle_{H}=0 \text { if } n \neq m
$$

where $\langle\cdot, \cdot\rangle_{H}$ is the inner-product on $H$.

Some unexpected facts about nuclear spaces are connected with the following simple observation:

Fact 2. Let $j: H \rightarrow K$ be Hilbert-Schmidt map between Hilbert spaces. Then there is a sequence of points in $H$ which does not converge in $H$ but whose image by $j$ in $K$ is convergent.

Proof. An orthonormal sequence $e_{1}, e_{2}, \ldots$ in $H$ is not Cauchy and hence not convergent, but the sequence $\left(j\left(e_{n}\right)\right)_{n \geq 1}$ converges to 0 in $K$ because $\sum_{n=1}^{\infty}\left\|j\left(e_{n}\right)\right\|_{K}^{2}$ is convergent.

\subsection{Dual of a Nuclear Space}

A linear functional on $\mathcal{H}$ is continuous with respect to $\|\cdot\|_{p}$ if and only if it extends to a (unique) continuous linear functional on $H_{p}$. Thus we may and will make the identification

$$
\mathcal{H}_{p}^{\prime} \simeq H_{-p} \stackrel{\text { def }}{=} H_{p}^{\prime}
$$

where the left side is the vector space of all linear functionals on $\mathcal{H}$ that are continuous with respect to the norm $\|\cdot\|_{p}$. To stress that we treat the dual space $H_{p}^{\prime}$ as a Hilbert space, i.e., having the strong topology, we denote it by $H_{-p}$; the norm on this space can also be obtained as

$$
\left\|x^{\prime}\right\|_{-p}=\sup _{x \in H_{p} ;\|x\|_{p} \leq 1}\left|\left\langle x^{\prime}, x\right\rangle\right|
$$

The topological dual of $\mathcal{H}$ is

$$
\mathcal{H}^{\prime}=\bigcup_{p=0}^{\infty} \mathcal{H}_{p}^{\prime} \supset \cdots \supset H_{2}^{\prime} \supset H_{1}^{\prime} \supset H_{0}^{\prime} \simeq H_{0}
$$

where we are viewing each $H_{p}^{\prime}$ as $\mathcal{H}_{p}^{\prime}$.

It is readily checked that the inclusion $H_{p}^{\prime} \rightarrow H_{p+1}^{\prime}$ is the adjoint of the inclusion map $H_{p+1} \rightarrow H_{p}$ and is thus also Hilbert Schmidt.

In addition to the weak and strong topologies, there is also the inductive limit topology on $\mathcal{H}^{\prime}$, which is the largest locally convex topology for which the inclusions $H_{p}^{\prime} \rightarrow \mathcal{H}^{\prime}$ are all continuous.

Fact 3. The strong topology and the inductive limit topology on $\mathcal{H}^{\prime}$ are the same. 
For a proof see [16].

\section{Balls and Cubes}

In this section, $p$ denotes an element of $\{0,1,2,3, \ldots\}$. We work, as before, with the space $\mathcal{H}$, equipped with inner-products $\langle\cdot, \cdot\rangle_{p}$, with the Hilbert-Schmidt condition explained after (9).

\subsection{Open and Closed Balls}

Letting $R$ denote any positive real number, we examine properties of the 'open' balls

$$
B_{p}(R)=\left\{y \in H_{p}:\|y\|_{p}<R\right\}
$$

and the "closed" balls

$$
D_{p}(R)=\left\{y \in H_{p}:\|y\|_{p} \leq R\right\}
$$

for every $p \in\{0,1,2, \ldots\}$. Any open or closed ball in a normed linear space is clearly bounded.

Fact 4. For all $p \in\{0,1,2, \ldots\}, B_{p}(R) \cap \mathcal{H}$ is open in $\mathcal{H}$.

Proof. The set $B_{p}(R) \cap \mathcal{H}$ is in fact just the open $R$-ball in the norm $\|\cdot\|_{p}$ on $\mathcal{H}$ and hence is in $\tau_{p}$, and therefore also in the topology $\tau$ on $\mathcal{H}$.

Fact 5. The set $B_{p}(R) \cap \mathcal{H}$ is not bounded in $\|\cdot\|_{p+1}$-norm. It is not bounded in $\mathcal{H}$.

Proof. Let $v_{1}, v_{2}, \ldots$ be an orthonormal basis of $H_{p+1}$ lying inside $\mathcal{H}$ (a maximal $\langle\cdot, \cdot\rangle_{p+1}$-orthonormal set in $\mathcal{H}$, which is dense inside $H_{p+1}$, is necessarily also maximal in $H_{p+1}$ and is hence an orthonormal basis of $\left.H_{p+1}\right)$. Then, since the inclusion $H_{p+1} \rightarrow H_{p}$ is Hilbert-Schmidt, the sum $\sum_{n=1}^{\infty}\left\|v_{n}\right\|_{p}^{2}$ is finite, and so the lengths $\left\|v_{n}\right\|_{p}$ tend to 0 . Thus the vectors

$$
\frac{R}{2}\left\|v_{n}\right\|_{p}^{-1} v_{n}
$$

which are all in $B_{p}(R)$, have $\langle\cdot, \cdot\rangle_{p+1}$-norm going to $\infty$. In particular, $B_{p}(R) \cap \mathcal{H}$ is not bounded in $H_{p+1}$. Consequently $B_{p}(R) \cap \mathcal{H}$ is also not bounded in $\mathcal{H}$.

The next observation provides some compact sets in $\mathcal{H}$.

Fact 6. The set $\bigcap_{p \geq 0} D_{p}\left(r_{p}\right)$ is compact in $\mathcal{H}$, for any $r_{1}, r_{2}, \ldots \in(0, \infty)$.

Proof. A closed ball $D_{p}(r) \cap \mathcal{H}$ is closed in the topology $\tau_{p} \subset \tau$, and so it is a closed subset of $\mathcal{H}$. If $W$ is a neighborhood of 0 in $\mathcal{H}$, then there is a $q \in\{0,1,2, .$.$\} and an r \in(0, \infty)$ such that $B_{q}(r) \subset W$; hence, $\bigcap_{p \geq 0} D_{p}\left(r_{p}\right) \subset t B_{q}(r) \subset t W$ if $t>r_{q} / r$. Thus, $\bigcap_{p \geq 0} D_{p}\left(r_{p}\right)$ is both closed and bounded and hence, by the Heine-Borel property proved below in Fact 14 , it is compact in $\mathcal{H}$

We turn to balls in the dual spaces $H_{p}^{\prime}$ and $\mathcal{H}^{\prime}$. Unless stated otherwise, we equip each Hilbert space dual $H_{-p} \simeq \mathcal{H}_{p}^{\prime}$ with the strong topology, which is the same as the Hilbert-space topology.

Fact 7. The set $\mathcal{H}^{\prime}$, with any topology making it a topological vector space, contains no non-empty open subset lying entirely inside $\mathcal{H}_{p}^{\prime}$, for $p \in\{0,1,2, \ldots\}$. More generally, in a topological vector space a proper subspace has empty interior.

Proof. If a proper subspace $Y$ of a topological vector space $X$ contains an open set $U$ with a point $y$ lying in $U$, then $-y+U$ is a neighborhood of 0 in $X$ lying entirely inside $Y$. Then the union of all multiples of $U-y$ would be all of $X$, and hence $Y$ would be all of $X$. 
Fact 8. The set $B_{-1}(R) \cap H_{0}$ is open in $H_{0}$. More generally, $B_{-q}(R) \cap H_{-p}$ is open in $H_{-p}$ for $p<q$, with $p, q \in\{0,1,2, \ldots\}$.

Proof. The inclusion $j: H_{q} \rightarrow H_{p}$ induces the adjoint $j^{*}: H_{-p} \rightarrow H_{-q}$ which is also continuous. So $\left(j^{*}\right)^{-1}\left(B_{-q}(R)\right)$ is open in $H_{-p}$. Identifying the dual spaces $H_{-p}$ and $\mathcal{H}_{p}^{\prime} \subset \mathcal{H}^{\prime}$, the map $j^{*}$ : $\mathcal{H}_{p}^{\prime} \rightarrow \mathcal{H}_{q}^{\prime}$ is just the inclusion map. Thus $\left(j^{*}\right)^{-1}\left(B_{-q}(R)\right)$ is the part of the ball $B_{-1}(R)$ that lies inside $H_{-p}$; that is, $\left(j^{*}\right)^{-1}\left(B_{-q}(R)\right)=B_{-q}(R) \cap H_{-p}$, which is thus open in $H_{-p}$.

Fact 9. The set $B_{0}(R)$ is not open in $H_{-1}$ in the strong (Hilbert) topology. More generally, $B_{-p}(R)$ is not strongly open in $H_{-q}$ for $p, q \in\{0,1,2, \ldots\}$ with $p<q$.

Proof. Note that $H_{-p}$ is a proper subspace of $H_{-q}$ for any $p<q$ by (17). By Fact $7, H_{-p}$ must have an empty interior in $H_{-q}$. Since $B_{-p}(R) \subset H_{-p}, B_{-p}(R)$ must also have an empty interior in $H_{-q}$. Thus $B_{-p}(R)$ is not open in $H_{-q}$.

Fact 10. The set $D_{-p}(R)$ is weakly, and hence also strongly, closed in $\mathcal{H}^{\prime}$. If $p, q \in\{0,1,2, \ldots\}$, with $q>p$, and $R \in(0, \infty)$, then $D_{-p}(R)$ is compact in the Hilbert-space $H_{q}^{\prime}$. Moreover, $D_{-p}(R)$ is strongly, and hence weakly, compact as well as sequentially compact in $\mathcal{H}^{\prime}$.

Proof. First, since $\|f\|_{-p}=\sup _{x \in H_{p},\|x\|_{p} \leq 1}|\langle f, x\rangle|$ we have

$$
D_{-p}(R)=\bigcap_{x \in H_{p},\|x\|_{p} \leq 1}\left\{f \in \mathcal{H}^{\prime} ;|\langle f, x\rangle| \leq R\right\} .
$$

Thus $D_{-p}(R)$ is the the intersection of weakly closed sets and is thus weakly closed. It follows that $D_{-p}(R)$ is also strongly closed in $\mathcal{H}^{\prime}$.

The inclusion map $H_{p+1} \rightarrow H_{p}$ being Hilbert-Schmidt, so is the adjoint inclusion $H_{-p} \rightarrow H_{-}(p+1)$, and thus $D_{-p}(R)$ is compact inside $H_{p+1}^{\prime}$ with the strong (Hilbert-space) topology. By continuity of the inclusion map $H_{q}^{\prime} \rightarrow \mathcal{H}^{\prime}$ it follows that $D_{-p}(R)$ is compact in $H_{q}^{\prime}$ with respect to the strong topology.

Continuity of the inclusion map $H_{p+1}^{\prime} \rightarrow \mathcal{H}^{\prime}$ also implies that $D_{-p}(R)$ is compact in $\mathcal{H}^{\prime}$ with respect to the strong topology.

Since $D_{-p}(R)$ is compact in the metric space $H_{q}^{\prime}$ it is sequentially compact in $H_{q}^{\prime}$, i.e., any sequence on $D_{-p}(R)$ has a subsequence which is convergent in $H_{q}^{\prime}$. From continuity of the inclusion $H_{q}^{\prime} \rightarrow \mathcal{H}^{\prime}$ it follows that such a subsequence also converges in $\mathcal{H}^{\prime}$. Thus, $D_{-p}(R)$ is strongly, and hence also weakly, sequentially compact in $\mathcal{H}^{\prime}$.

\subsection{Cubes}

We use the the term "cube" for what might more properly be called a "box" in a linear space, bounded by "walls". For the following observation about small closed cubes with nonempty interior we focus on a nuclear space $\mathcal{H}$ for which there is an orthonormal basis $\left(e_{n}\right)_{n \geq 1}$ of $H_{0}$, the vectors of which all lie in $\mathcal{H}$ and are orthogonal within each $H_{n}$, with $1 \leq\left\|e_{n}\right\|_{p}=\left\|e_{n}\right\|_{1}^{p}$ for all $n \geq 1$ and $p \geq 0$. This is the structure in many applications, including the Schwartz space (see Section 8).

Fact 11. Suppose $e_{1}, e_{2}, \ldots$ is an orthonormal basis of $H_{0}$ which lies in $\mathcal{H}$, and assume that the vectors $\lambda_{n}^{-p} e_{n}$ form an orthonormal basis of $H_{p}$, where $\lambda_{n}=\left\|e_{n}\right\|_{1}$. Assume also that

$$
1 \leq \lambda_{1}<\lambda_{2}<\cdots \quad \text { and } \quad \sum_{n=1}^{\infty} \lambda_{n}^{-2}<\infty .
$$


Let $C$ be the closed cube in $\mathcal{H}^{\prime}$ given by

$$
C=\left\{x^{\prime} \in \mathcal{H}^{\prime}:\left|\left\langle x^{\prime}, e_{n}\right\rangle\right| \leq \lambda_{n}^{n} \text { for all } n \in\{1,2,3, \ldots\}\right\}
$$

Then $C$ contains a strong neighborhood of 0 and hence is not compact. However, $C \cap F$ is compact for every finite-dimensional subspace of $H_{0}$.

Proof. Let $x^{\prime} \in B_{-p}\left(\epsilon_{p}\right)$, for some $p \in\{0,1,2,3, \ldots\}$, and $\epsilon_{p}>0$. Then, for every $n$,

$$
\left|\left\langle x^{\prime}, e_{n}\right\rangle\right| \leq \epsilon_{p}\left\|e_{n}\right\|_{p} .
$$

To make this $\leq \lambda_{n}^{n}$, we should take

$$
\epsilon_{p} \leq\left\|e_{n}\right\|_{p}^{-1} \lambda_{n}^{n}=\lambda_{n}^{n-p}
$$

and this should hold for all $n \in\{1,2,3, \ldots\}$. Thus, we could take $\epsilon_{0}=1$, and, for $p \in\{1,2,3, \ldots\}$ :

$$
\epsilon_{p}=\min \{\lambda_{1}^{1-p}, \lambda_{2}^{2-p}, \ldots, \underbrace{\lambda_{p}^{p-p}}_{1}\}
$$

wherein we have dropped all the later terms $\lambda_{n}^{n-p}$ with $n>p$, as these are all $\geq 1$. Thus, with this choice of $\epsilon_{p}$, the cube

$$
C=\left\{x^{\prime} \in \mathcal{H}^{\prime}:\left|\left\langle x^{\prime}, e_{n}\right\rangle\right| \leq \lambda_{n}^{n} \text { for all } n \in\{1,2,3, \ldots\}\right\}
$$

contains each open ball $B_{-p}\left(\epsilon_{p}\right)$ and hence also the convex hull of their union, which is a neighborhood of 0 in the inductive limit topology (see [6], Chapter V, Section 2).

Now consider a finite-dimensional subspace $F$ of $H_{0} \simeq H_{0}^{\prime} \subset \mathcal{H}^{\prime}$, and $v^{\prime}$ a point in $F \cap C$. Then

$$
\left|\hat{e}_{n}\left(v^{\prime}\right)\right| \leq \lambda_{n}^{n}
$$

for all $n \in\{1,2,3, \ldots\}$. Let us assume, for the moment, that there is an orthonormal basis $f_{1}, \ldots, f_{d}$ of $F \subset H_{0}$ and $a$ fixed $r \in\{1,2,3, \ldots\}$ such that each $\left\langle f_{j}, \cdot\right\rangle_{0}$ on $F$ is a linear combination of the functionals $\hat{e}_{1}, \ldots, \hat{e}_{r}$, say

$$
\left\langle f_{j},\right\rangle_{0}=\sum_{n=1}^{r} a_{j n} \hat{e}_{n} .
$$

Then

$$
\left|\left\langle f_{j}, v^{\prime}\right\rangle\right| \leq R_{j} \stackrel{\text { def }}{=} \sum_{n=1}^{r}\left|a_{j n}\right| \lambda_{n}^{n}<\infty .
$$

Hence,

$$
\left\|v^{\prime}\right\|_{0}^{2} \leq R_{1}^{2}+\cdots+R_{d}^{2}<\infty
$$

This shows that $C \cap F$ is a bounded subset of $F$. Since $C$ is weakly closed and so is any finite-dimensional subspace, $C \cap F$ is weakly closed in $\mathcal{H}^{\prime}$ and hence weakly closed in the induced topology on $F$; but on the finite-dimensional subspace $F$ there is only one topological vector space structure, and so $C \cap F$ is closed. Being closed and bounded in $F$, it is compact.

It remains to prove the algebraic statement assumed earlier. Suppose that the algebraic linear span of the functionals $\hat{e}_{n}$ on $F$ is not the entire dual $F^{*}$ of $F$; then they span a proper subspace of $F^{*}$ and hence there is a non-zero vector $f \in F$ on which they all vanish, but this would then be a vector in $H_{0}$ whose inner-product with every $e_{n}$ is 0 and hence $f$ would have to be 0 . Thus the algebraic linear span of the functionals $\hat{e}_{n}$ on $F$ is the entire dual $F^{*}$, and so, in particular, any element of $F^{*}$ is a finite linear combination of the functionals $\hat{e}_{n}$. This establishes the assumption made earlier. 


\section{Facts about the Nuclear Space Topology}

We work with an infinite-dimensional nuclear space $\mathcal{H}$ with structure as detailed in Section 3. While there are some treacherous features, such as the one in Fact 12, a nuclear space has some very convenient properties that make them almost as good as finite-dimensional spaces.

Fact 12. There is no non-empty bounded open set in $\mathcal{H}$.

Proof. Suppose $U$ is a bounded open set in $\mathcal{H}$ containing some point $y$. Then $U-y$ is a bounded open neighborhood of 0 in $\mathcal{H}$ and so contains some ball $B_{p}(R) \cap \mathcal{H}$, with $p \in\{0,1,2, \ldots\}$. By Fact 5 this is impossible.

Fact 13. The topology on $\mathcal{H}$ is metrizable but not normable.

Proof. If there were a norm then the unit ball in the norm would be a bounded neighborhood of 0 , contradicting Fact 12 . The translation-invariant metric on $\mathcal{H}$ given by

$$
d(x, y)=\sum_{p=0}^{\infty} 2^{-p} \min \left\{1,\|x-y\|_{p}\right\}
$$

induces the topology on $\mathcal{H}$.

One immediate consequence of the preceding observations is that an infinite dimensional Banach space is not a nuclear space.

If $f: X \rightarrow Y$ is a continuous linear map between topological vector spaces and $B$ is a bounded subset of $X$ then $f(B)$ is a bounded subset of $Y$, for if $V$ is a neighborhood of 0 in $Y$ then $B \subset t f^{-1}(V)=$ $f^{-1}(t V)$ for some scalar $t$ and hence $f(B) \subset t V$. This observation is used to prove the following useful fact about nuclear spaces.

Fact 14. The nuclear space $\mathcal{H}$ has the Heine-Borel property: every closed and bounded set is compact.

Proof. Let $B$ be a closed and bounded subset of $\mathcal{H}$. Take any $p \in\{0,1,2, .$.$\} . Since the inclusion$ $\mathcal{H} \rightarrow H_{p+1}$ is continuous, $B$ is bounded in $H_{p+1}$. Since the inclusion $H_{p+1} \rightarrow H_{p}$ is Hilbert-Schmidt it follows that $B$, as a subset of $H_{p}$, is contained in a compact set. Let $\left(x_{n}\right)_{n \geq 1}$ be a sequence of points in $B$. Then there is a subsequence $\left(x^{(p)}\right)_{n \geq 1}$ that converges in $H_{p}$. Applying the Cantor process of extracting repeated subsequences, there is a subsequence $\left(x_{n}^{\prime}\right)_{n \geq 1}$ that is convergent in $H_{p}$ for every $p \in\{0,1,2, \ldots\}$. By continuity of the inclusions $H_{p+1} \rightarrow H_{p}$ it follows that the limit is the same for every $p$, i.e., there is a point $y \in \bigcap_{p \geq 0} H_{p}=\mathcal{H}$, such that the subsequence $\left(x_{n}^{\prime}\right)$ converges to $y$ in all the $H_{p}$. If $V$ is a neighborhood of 0 in $\mathcal{H}$ then there is a $p \in\{0,1,2, \ldots\}$ and an $\epsilon>0$ such that $B_{p}^{\mathcal{H}}(\epsilon)=\left\{x \in \mathcal{H}:\|x\|_{p}<\epsilon\right\} \subset V$. Since $x_{n}^{\prime} \rightarrow y$ in $H_{p}$ we have $x_{n}^{\prime}-y \in B_{p}^{\mathcal{H}}(\epsilon) \subset V$ for large $n$, and so $x_{n}^{\prime} \rightarrow y$ in $\mathcal{H}$. Thus, a closed and bounded subset of $\mathcal{H}$ is sequentially compact; since $\mathcal{H}$ is metrizable, sequential compactness is equivalent to compactness for any subset of $\mathcal{H}$.

\section{Facts about the Dual Topologies}

We turn now to the dual of an infinite-dimensional nuclear space $\mathcal{H}$, and continue with the notation explained earlier in the context of Section 3.

Let us recall [11] the Banach-Steinhaus theorem (uniform boundedness principle): If $S$ is a non-empty set of continuous linear functionals on a complete, metrizable, topological vector space $X$, and if, for each $x \in X$ the set $\{f(x): f \in S\}$ is bounded, then for every neighborhood $W$ of 0 in the scalars, there is a neighborhood $U$ of 0 in $X$ such that $f(U) \subset W$ for all $f \in S$. As consequence, if $f_{1}, f_{2}, \ldots \in X^{\prime}$ are such that $f(x)=\lim _{n \rightarrow \infty} f_{n}(x)$ exists for all $x \in X$ then $f$ is in $X^{\prime}$. 
The following facts in this section are standard and can be found in any of $[5-8,10]$, among other places.

Fact 15. If $X$ is a complete, metrizable topological vector space, then the dual space $X^{\prime}$ is complete with respect to both strong and weak topologies. In particular, the dual $\mathcal{H}^{\prime}$ of a nuclear space $\mathcal{H}$ is complete with respect to both the strong and weak topologies.

Proof. Let $\mathcal{A}$ be a non-empty collection of subsets of $X$, closed under finite unions and whose union is all of $X$. Let $\tau_{\mathcal{A}}$ be the topology on $X$ whose open sets are unions of translates of sets of the form

$$
B(A ; \epsilon)=\left\{x^{\prime} \in X^{\prime}: \sup _{x \in A}\left|\left\langle x^{\prime}, x\right\rangle\right|<\epsilon\right\}
$$

with $A$ running over $\mathcal{A}$ and $\epsilon$ over $(0, \infty)$. If $\mathcal{A}$ is the set of all finite subsets of $X$ then $\tau_{\mathcal{A}}$ is the weak topology; if $\mathcal{A}$ is the set of all bounded subsets of $X$ then $\tau_{\mathcal{A}}$ is the strong topology.

Suppose $\left(f_{n}\right)_{n \geq 1}$ is Cauchy sequence in $X^{\prime}$ for the topology $\tau_{\mathcal{A}}$. This means that, for any $A \in \mathcal{A}$, the sequence of functions $f_{n} \mid A$ is uniformly Cauchy, and hence uniformly convergent, for every $A \in \mathcal{A}$. Let $f(x)=\lim _{n \rightarrow \infty} f_{n}(x)$. By Banach-Steinhaus, $f$ is continuous. A $\tau_{\mathcal{A}}$-neighborhood $U$ of $f$ contains a set of the form $f+B(A ; \epsilon)$, and the uniform convergence $f_{n} \rightarrow f$ on $A$ implies that $f_{n}-f \in B(A ; \epsilon)$ for large $n$, and so $f_{n}-f \in U$ for large $n$. Thus, $f_{n} \rightarrow f$ in $\tau_{\mathcal{A}}$.

Fact 16. Any weakly open neighborhood of 0 in the dual of an infinite dimensional locally convex topological vector space contains an infinite-dimensional subspace.

Proof. If $Y$ is a vector space over a field $F$, and $\hat{x}_{1}, \ldots, \hat{x}_{N}: Y \rightarrow F$ are linear functionals, then the mapping

$$
Y \rightarrow F^{N}: y \mapsto\left(\hat{x}_{1}(y), \ldots, \hat{x}_{N}(y)\right)
$$

has kernel infinite-dimensional if $Y$ is infinite-dimensional. We apply this to $Y=X^{\prime}$. A weakly open neighborhood of 0 in $X^{\prime}$ contains contains a set of the form

$$
B=\left\{f \in X^{\prime}: \hat{x}_{1}(f) \in W_{1}, \ldots, \hat{x}_{N}(f) \in W_{N}\right\},
$$

for some $x_{1}, \ldots, x_{N} \in X$, and open neighborhoods $W_{1}, \ldots, W_{N}$ of 0 in the scalars, and so $B$ contains the $\operatorname{ker}\left(\hat{x}_{1}, \ldots, \hat{x}_{N}\right)$.

The weak dual of an infinite-dimensional Banach space is not metrizable. In a similar vein there is the following negative result:

Fact 17. The weak topology and the strong topology on $\mathcal{H}^{\prime}$ are not metrizable.

Proof. Consider the nuclear space $\mathcal{H}$ with topology generated by norms $\|\cdot\|_{p}$ for $p \in\{0,1,2, \ldots\}$ as in Section 3. Let $D_{p}(R)$ be the subset of $\mathcal{H}$ given by the closed ball of radius $R$, center 0 , for the $\|\cdot\|_{p}$-norm, and let $D_{p}^{\prime}(R)$ be the set of all linear functionals $f$ in $\mathcal{H}^{\prime}$ which map $D_{p}(1)$ into scalars of magnitude $\leq R$. Each set $D_{p}^{\prime}(R)$, being the intersection of weakly closed sets, is a weakly closed set, and hence also strongly closed, in $\mathcal{H}^{\prime}$. The union of the sets $D_{p}^{\prime}(n)$ with $p \in\{0,1,2, \ldots\}$ and $n \in\{1,2, \ldots\}$ is all of $\mathcal{H}^{\prime}$. Now $D_{p}^{\prime}(n)$ lies in the proper subspace $H_{-p}$ of $\mathcal{H}^{\prime}$, and so has empty interior in any topology on $\mathcal{H}^{\prime}$ which makes $\mathcal{H}^{\prime}$ a topological vector space by Fact 7 . Thus, $\mathcal{H}^{\prime}$, which is a complete topological vector space with respect to both weak and strong topologies, is the countable union of nowhere dense sets, and hence the weak and strong topologies on $\mathcal{H}^{\prime}$ are not metrizable.

In a sense, sequences do not detect the difference between the weak and strong topologies on the dual space: 
Fact 18. A sequence in $\mathcal{H}^{\prime}$ is weakly convergent if and only if it is strongly convergent.

Proof. Since the weak topology is contained in the strong topology, strong convergence implies weak convergence. For the converse, let $\left(x_{n}^{\prime}\right)_{n \geq 1}$ be a sequence in the dual $\mathcal{H}^{\prime}$ of the nuclear space $\mathcal{H}$, converging weakly to $x^{\prime} \in \mathcal{H}^{\prime}$. By Banach-Steinhaus, $\left\{x_{1}^{\prime}, x_{2}^{\prime}, x_{3}^{\prime}, \ldots\right\}$ is a uniformly continuous set of functions on $\mathcal{H}$ and hence, as may be checked, the convergence $x_{n}^{\prime} \rightarrow x^{\prime}$ is uniform on compact subsets of $\mathcal{H}$. The closure $\bar{D}$ of the bounded set $D$ is bounded (see Rudin [11] (Theorem 1.13(f))) and hence also compact by the Heine-Borel property for nuclear spaces Fact 14 . So the sequence $\left(x_{n}^{\prime}\right)_{\geq 1}$ is uniformly convergent on $\bar{D}$, and hence also on $D$, and therefore it is strongly convergent as a sequence in $\mathcal{H}^{\prime}$.

\subsection{Bounded and Compact Sets in the Dual of Nuclear Space}

We now examine bounded and compact sets in $\mathcal{H}^{\prime}$. In particular, we will see that weakly and strongly bounded sets are one in the same. The same goes for weakly and strongly compact sets.

Fact 19. A set $B \in \mathcal{H}^{\prime}$ is strongly bounded if and only if $B$ is bounded on each bounded set in $\mathcal{H}$.

Proof. Let $B \subset \mathcal{H}^{\prime}$ be strongly bounded and let $D$ be a bounded set of $\mathcal{H}$. Consider the local base set of $\mathcal{H}^{\prime}$ (for the strong topology) given by

$$
B(D ; 1)=\left\{f \in \mathcal{H}^{\prime} ; \sup _{x \in D}|\langle f, x\rangle|<1\right\} .
$$

Since $B$ is bounded there exists an $\alpha>0$ such that $B \subset \alpha B(D ; 1)$ or, equivalently, $\frac{1}{\alpha} B \subset B(D ; 1)$. Then for any $f \in B$ we have that $\frac{f}{\alpha} \in B(D ; 1)$. Thus $|\langle f, x\rangle| \leq \alpha$ for any $x \in D$. Therefore $B$ is bounded on the set $D$.

Suppose $B$ is bounded on each bounded set $D \subset \mathcal{H}$. Consider the open set $B(D ; \epsilon)$ containing 0 in $\mathcal{H}^{\prime}$. By hypothesis, $\sup _{f \in B, x \in D}|\langle f, x\rangle|=M<\infty$. So, for any $f \in B$ we have that $\left|\left\langle\frac{\epsilon f}{M+1}, x\right\rangle\right|<\epsilon$ when $x \in D$. Therefore $\frac{\epsilon}{M+1} B \subset B(D ; 1)$ or, equivalently, $B \subset \frac{M+1}{\epsilon} B(D ; \epsilon)$. Hence $B$ is bounded.

Fact 20. $A$ set $B$ in $\mathcal{H}^{\prime}$ is strongly bounded if and only if there exists $p$ such that $B$ is bounded on $B_{p}^{\mathcal{H}}\left(\frac{1}{p}\right)=\left\{x \in \mathcal{H}:\|x\|_{p}<\frac{1}{p}\right\}$.

Proof. Consider the local base for $\mathcal{H}$ given by

$$
B_{1}^{\mathcal{H}}(1) \supset B_{2}^{\mathcal{H}}\left(\frac{1}{2}\right) \supset B_{3}^{\mathcal{H}}\left(\frac{1}{3}\right) \supset \cdots
$$

By contradiction suppose that $B$ is not bounded on $B_{p}^{\mathcal{H}}\left(\frac{1}{p}\right)$ for any integer $p>0$. Then for every $p$ there exists $x_{p} \in B_{p}^{\mathcal{H}}\left(\frac{1}{p}\right)$ and a $f_{p} \in B$ such that $\left|\left\langle f_{p}, x_{p}\right\rangle\right|>p$. By construction, the sequence $\left(x_{p}\right)_{p=1}^{\infty}$ goes to 0 in $\mathcal{H}$ and thus must be bounded. So there must exists a positive number $M$ such that $\left|\left\langle f, x_{p}\right\rangle\right| \leq M$ for all $f \in B$ and all $p \in\{1,2,3, \cdots\}$. This contradicts the way by which $f_{p}$ and $x_{p}$ were chosen.

Conversely, let $B \subset \mathcal{H}^{\prime}$ be bounded on some $B_{p}^{\mathcal{H}}\left(\frac{1}{p}\right) \subset \mathcal{H}$. Take a bounded set $D$ in $\mathcal{H}$. Then $D \subset$ $\alpha B_{p}^{\mathcal{H}}\left(\frac{1}{p}\right)$ for some $\alpha>0$. Thus $B$ is bounded on $D$ and is therefore bounded on $\mathcal{H}^{\prime}$.

The following result provides us with a way of studying bounded sets in $\mathcal{H}^{\prime}$ in terms of the norms $\|\cdot\|_{-p}$ on $H_{-p}$.

Fact 21. A set $B \subset \mathcal{H}^{\prime}$ is strongly bounded if and only if there exists an integer $p \geq 0$ such that $B \subset H_{-p}$ and $B$ is bounded in the norm $\|\cdot\|_{-p}$ on $H_{-p}$. 
Proof. The converse direction is the easier of the two. If $B \subset H_{-p}$ and $\|f\|_{-p}<M$ for all $f \in B$, then $B$ is bounded on $B_{p}(1)$ and consequently bounded on $B_{p}^{\mathcal{H}}(1 / p)$. Thus Fact 20 applies to tell us that $B$ is strongly bounded.

Now for the forward direction: suppose $B$ is a strongly bounded set in $\mathcal{H}^{\prime}$. Then by Fact 20 there exists an integer $p \geq 0$ such that $B$ is bounded on the set $B_{p}^{\mathcal{H}}\left(\frac{1}{p}\right)=\left\{x \in \mathcal{H} ;\|x\|_{p}<\frac{1}{p}\right\}$. That is, there is an $M<\infty$ such that $|\langle f, x\rangle| \leq M$ for all $f \in B$ and all $x \in B_{p}^{\mathcal{H}}\left(\frac{1}{p}\right)$.

Consider the set in $H_{p}$ given by $B_{p}\left(\frac{1}{p}\right)=\left\{x \in H_{p} ;\|x\|_{p}<\frac{1}{p}\right\}$. Since $\mathcal{H}$ is dense in $H_{p}$ we have that

$$
\sup _{f \in B, x \in B_{p}(1 / p)}|\langle f, x\rangle| \leq M .
$$

From the above we see that for any $f \in B$ and any nonzero vector $x \in H_{p}$ we have

$$
\left|\left\langle f, \frac{x}{\|x\|_{p}(p+1)}\right\rangle\right| \leq M
$$

Therefore $\|f\|_{-p} \leq(p+1) M$ for any $f \in B$. Thus for any $f \in B$ we have that $f \in H_{-p}$ and $\|f\|_{-p} \leq(p+1) M$.

Fact 22. A set $B \subset \mathcal{H}^{\prime}$ is weakly bounded if and only if $B$ is strongly bounded.

Proof. The converse direction is straightforward: Assuming a set $B$ is strongly bounded, then it must also be weakly bounded since the strong topology on $\mathcal{H}^{\prime}$ is finer than the weak topology on $\mathcal{H}^{\prime}$.

For the forward direction, we let $B \subset \mathcal{H}^{\prime}$ be a weakly bounded set. Define the set $C \subset \mathcal{H}$ as follows:

$$
C=\{x \in \mathcal{H} ;|\langle f, x\rangle| \leq 1 \text { for all } f \in B\}=\bigcap_{f \in B}\{x \in \mathcal{H} ;|\langle f, x\rangle| \leq 1\} .
$$

Note that $C$ is the intersection of convex, closed, and balanced sets. Therefore $C$ is also a convex, closed, and balanced set.

Also note that $C$ is absorbent: Take $x \in \mathcal{H}$. Since $B$ is weakly bounded there must exist $M>0$ such that $B \subset M N_{1}(x)$ where $N_{1}(x)=\left\{f \in \mathcal{H}^{\prime} ;|\langle f, x\rangle|<1\right\}$. For any $\alpha$ satisfying $|\alpha| \geq M$ we have that $|\langle f, x\rangle| \leq M \leq|\alpha|$ for all $f \in B$. Hence $\frac{x}{\alpha} \in C$ or equivalently $x \in \alpha C$. This gives us that $C$ is absorbing.

Since $C$ is absorbing we have that $\mathcal{H}=\bigcup_{n=1}^{\infty} n C$. Knowing that $\mathcal{H}$ is a complete metric space (Facts 13 and 15) we can apply the Baire category theorem to see that $C$ is not nowhere dense. Thus the interior of $C, C^{\circ}$, is not empty. Take $x_{0} \in C^{\circ}$ and a set $B_{p}^{\mathcal{H}}(\epsilon)=\left\{x \in \mathcal{H}:\|x\|_{p}<\epsilon\right\}$ such that $x_{0}+B_{p}^{\mathcal{H}}(\epsilon) \subset C^{\circ}$. Because $C$ is balanced we have

$$
-x_{0}-B_{p}^{\mathcal{H}}(\epsilon) \subset C
$$

and since $-B_{p}^{\mathcal{H}}(\epsilon)=B_{p}^{\mathcal{H}}(\epsilon)$ we have

$$
-x_{0}+B_{p}^{\mathcal{H}}(\epsilon) \subset C .
$$

Now $C$ is convex with $-x_{0}+B_{p}^{\mathcal{H}}(\epsilon) \subset C$ and $x_{0}+B_{p}^{\mathcal{H}}(\epsilon) \subset C$. So we must have that the convex hull of $\left(-x_{0}+B_{p}^{\mathcal{H}}(\epsilon)\right) \cup\left(x_{0}+B_{p}^{\mathcal{H}}(\epsilon)\right)$ is contained in $C$. But this convex hull contains $B_{p}^{\mathcal{H}}(\epsilon)$ : Observe, for any $x \in B_{p}^{\mathcal{H}}(\epsilon)$ we have that

$$
x=\frac{1}{2}\left(x_{0}+x\right)+\frac{1}{2}\left(-x_{0}+x\right) .
$$

Therefore $B_{p}^{\mathcal{H}}(\epsilon) \subset C$.

Since $B$ is bounded on $C$ and $B_{p}^{\mathcal{H}}(\epsilon) \subset C$ we have that $B$ is bounded on $B_{p}^{\mathcal{H}}(\epsilon)$ and is thus strongly bounded. 
Fact 23. A set $K \subset \mathcal{H}^{\prime}$ is weakly compact if and only if $K$ is strongly compact.

Proof. Since the strong topology is finer than the weak topology, this immediately gives us that if $K$ is strongly compact, then $K$ is weakly compact.

For the other direction suppose $K$ is weakly compact. Then $K$ is weakly bounded. Thus we can apply Fact 22 to obtain that $K$ is strongly bounded. By Fact 21 there exists an integer $p \geq 0$ and a real number $R$ such that $K \subset H_{-p}$ and $\|x\|_{-p} \leq R$ for all $x$ in $K$.

Since $K$ is weakly compact in $\mathcal{H}^{\prime}, K$ is also weakly closed in $\mathcal{H}^{\prime}$ Therefore, because the inclusion map is continuous we have $i_{p}^{\prime}-1(K)=K$ is closed in $H_{-p}$. Also, using that $K \subset H_{-p} \subset H_{-p-1}$ we have $i_{p+1}^{\prime}-1(K)=K$ is closed in $H_{-p-1}$, where again we use the continuity of the inclusion map.

Because $K$ is bounded in $H_{-p}$, we get $I_{p, p+1}^{\prime}(K)=K$ has compact closure in $H_{-p-1}$. (Here we have used that the inclusion map from $H_{-p}$ to $H_{-p-1}$ is Hilbert-Schmidt and is thus a compact operator). However, we have seen $K$ is closed in $H_{-p-1}$. Hence $K$ is compact in $H_{-p-1}$. The continuity of the inclusion map $i_{p+1}^{\prime}$ from $H_{-p-1}$ to $\mathcal{H}^{\prime}$ gives us the continuous image $i_{p+1}^{\prime}(K)=K$ is compact in $\mathcal{H}^{\prime}$.

\subsection{The Dual Space is Sequential}

Now we examine the viability of using sequences to study the dual space $\mathcal{H}^{\prime}$. It turns out that the topology on the dual space $\mathcal{H}^{\prime}$ does allow us to study topological properties such as closure and continuity in terms of sequences. We have the following result:

Fact 24. The dual space $\mathcal{H}^{\prime}$, equipped with the strong/inductive limit topology, is a sequential space: if a subset $C$ of $\mathcal{H}^{\prime}$ is such that every convergent sequence in $C$ has limit lying in $C$ then $C$ is closed.

To prove this result we make use of the following Lemma about Hilbert spaces that is fairly straightforward to prove.

Lemma 1. Let $H$ be a Hilbert space. Suppose $K$ is a compact set and $C$ is a closed set in $H$. There exists $\epsilon>0$ and corresponding closed (or open) ball $D(\epsilon)=\{x \in H ;\|x\| \leq \epsilon\}$ such that

$$
(K+D(\epsilon)) \cap C=\varnothing .
$$

Proof. (of Fact 24) Suppose $C$ is sequentially closed in $\mathcal{H}^{\prime}$. We show $C$ is closed by selecting a point outside of $C$ and showing that this point is also not in the closure of $C$. Let $f \notin C$. Because $f \in \mathcal{H}^{\prime}$, $f \in H_{-p}$ for some integer $p \geq 0$. Since $C$ is sequentially closed, then using the inclusion map $i_{p}^{\prime}: H_{-p} \rightarrow \mathcal{H}^{\prime}$ we have $i_{p}^{\prime-1}(C)$ is sequentially closed in $H_{-p}$. Thus $i_{p}^{\prime-1}(C)$ is closed in the Hilbert space $H_{-p}$. Since $f \notin i_{p}^{\prime}-1(C)$ there exists $\epsilon_{p}>0$ such that

$$
i_{p}^{\prime-1}(C) \cap\left(f+D_{-p}\left(\epsilon_{p}\right)\right)=\varnothing
$$

where $D_{-p}\left(\epsilon_{p}\right)=\left\{f \in H_{-p} ;\|f\|_{-p} \leq \epsilon_{p}\right\}$.

Now consider the inclusion map $I_{p, p+1}^{\prime}: H_{-p} \rightarrow H_{-p-1}$. Then $I_{p, p+1}^{\prime}\left(D_{-p}\left(\epsilon_{p}\right)\right)$ is closed and compact in $H_{-p-1}$ (see Fact 8 ).

By Lemma 1 there exists $D_{-p-1}\left(\epsilon_{p+1}\right)=\left\{f \in H_{-p-1} ;\|f\|_{-p-1} \leq \epsilon_{p+1}\right\}$ such that

$$
\left(f+I_{p, p+1}^{\prime}\left(D_{-p}\left(\epsilon_{p}\right)\right)+D_{-p-1}\left(\epsilon_{p+1}\right)\right) \cap i_{-p-1}^{\prime-1}(C)=\varnothing .
$$

Repeating this process we obtain $\left\{\epsilon_{p+k}\right\}_{k=1}^{\infty}$ such that

$$
\left(f+I_{p, p+k}^{\prime}\left(D_{-p}\left(\epsilon_{p}\right)\right)+I_{p, p+k}^{\prime}\left(D_{-p-1}\left(\epsilon_{p+1}\right)\right)+\cdots+D_{-p-k}\left(\epsilon_{p+k}\right)\right) \cap i_{-p-1}^{\prime-1}(C)=\varnothing .
$$


Consider the set $N$ given by

$$
N=\bigcup_{k=0}^{\infty} I_{p, p+k}^{\prime}\left(D_{-p}\left(\epsilon_{p}\right)\right)+I_{p, p+k}^{\prime}\left(D_{-p-1}\left(\epsilon_{p+1}\right)\right)+\cdots+D_{-p-k}\left(\epsilon_{p+k}\right) .
$$

We now show

1. $N$ is convex:

Take $f, g \in N$. Recalling that $H_{-p} \subset H_{-q}$ when $q \geq p$ we have the union of sets making up $N$ is an increasing union. Thus there exists a $k$ such that

$$
f, g \in I_{p, p+k}^{\prime}\left(D_{-p}\left(\epsilon_{p}\right)\right)+I_{p, p+k}^{\prime}\left(D_{-p-1}\left(\epsilon_{p+1}\right)\right)+\cdots+D_{-p-k}\left(\epsilon_{p+k}\right) .
$$

Note that the above is a sum of convex sets and is consequently convex. Hence, for any $\alpha \in[0,1]$, we have $\alpha f+(1-\alpha) g$ is also in $I_{p, p+k}^{\prime}\left(D_{-p}\left(\epsilon_{p}\right)\right)+I_{p, p+k}^{\prime}\left(D_{-p-1}\left(\epsilon_{p+1}\right)\right)+\cdots+D_{-p-k}\left(\epsilon_{p+k}\right)$ and therefore also in $N$. So $N$ is convex.

2. $\quad N$ contains $\bigcup_{q=0}^{\infty} B_{-q}\left(\epsilon_{q}\right)$ where $\epsilon_{q}=\epsilon_{p}$ for $q \leq p$ :

For $f \in B_{-q}\left(\epsilon_{q}\right)$ we have $f \in \mathcal{H}^{\prime}$ and $\|f\|_{-q} \leq \epsilon_{q}$. When $q>p$ we have $q=p+k$ for some positive integer $k$ and thus $B_{q}\left(\epsilon_{q}\right) \subset D_{-p-k}\left(\epsilon_{p+k}\right) \subset N$. For $q \leq p$ we have $B_{-q}\left(\epsilon_{q}\right) \subset D_{-q}\left(\epsilon_{q}\right) \subset$ $D_{-p}\left(\epsilon_{p}\right) \subset N$ using that $\epsilon_{q}=\epsilon_{p}$ and $\|\cdot\|_{-p} \leq\|\cdot\|_{-q}$.

Therefore $N$ contains the convex hull of $\bigcup_{q=0}^{\infty} B_{q}\left(\epsilon_{q}\right)$ (where $\epsilon_{q}=\epsilon_{p}$ for $q \leq p$ ), which is a neighborhood of 0 in the inductive limit topology.

By construction we must have $(f+N) \cap C=\varnothing$. Thus $f$ is not a member of the closure of $C$. Hence $C$ is closed.

An argument very similar to the above can be used to demonstrate that $\mathcal{H}^{\prime}$ is a $k$-space. That is, $C \subset \mathcal{H}^{\prime}$ is closed if and only if $K \cap C$ is compact for any compact set $K \subset \mathcal{H}^{\prime}$.

\subsection{Separability}

We now examine the separability of the dual space $\mathcal{H}^{\prime}$.

Fact 25. The space $\mathcal{H}^{\prime}$ is separable when $\mathcal{H}^{\prime}$ is endowed with the strong, inductive limit, or weak topology.

Proof. We first need a candidate for a countable dense set. Since $\mathcal{H}^{\prime}=\bigcup_{p=0}^{\infty} H_{-p}$ and each $H_{-p}$ is a separable Hilbert space we let $Q_{p}$ be a countable dense subset of $H_{-p}$ and form the countable set

$$
Q=\bigcup_{p=0}^{\infty} Q_{p}
$$

We will show $Q$ is dense in $\mathcal{H}^{\prime}$.

Take $f \in \mathcal{H}^{\prime}$. Then $f \in H_{-p}$ for some $p$. Let $U$ be an open set containing $f$ in $\mathcal{H}^{\prime}$ ( $\mathcal{H}^{\prime}$ can be endowed with the weak, strong, or inductive limit topology). Then $-f+U$ is an open set about 0 . We know the inclusion maps from $i_{p}^{\prime}: H_{-p} \rightarrow \mathcal{H}^{\prime}$ are continuous when $\mathcal{H}^{\prime}$ has the weak, strong, or inductive limit topology. Therefore $i_{p}^{\prime-1}(-f+U)$ is an open set about 0 in $H_{-p}$ and as such there exists an $\epsilon>0$ such that

$$
B_{-p}(\epsilon) \subset i_{p}^{\prime-1}(-f+U)=-f+i_{p}^{-1}(U)
$$

or equivalently

$$
f+B_{-p}(\epsilon) \subset i_{p}^{\prime-1}(U)
$$

Since $Q_{p}$ is dense in $H_{-p}$ there exists $f_{p} \in Q_{p}$ such that

$$
\left\|f_{p}-f\right\|_{-p}<\epsilon
$$


That is, $f_{p} \in f+B_{-p}(\epsilon) \subset i_{p}^{\prime}-1(U)$. Thus $f_{p} \in U$ showing that $Q$ is dense in $\mathcal{H}^{\prime}$ and $\mathcal{H}^{\prime}$ is separable.

\subsection{First Countability (Lack Thereof)}

A topological space is said to be first countable if each point has countable local base. It is said to be second countable if the topology has a countable base. In this section we seek to determine if the dual space $\mathcal{H}^{\prime}$ is first countable. From previous results we know $\mathcal{H}^{\prime}$ is separable (Fact 25). Also, we saw that $\mathcal{H}^{\prime}$ can be constructed as the inductive limit of separable Hilbert spaces, which are first (and second) countable. Unfortunately though, $\mathcal{H}^{\prime}$ does not retain the property of first countability.

Fact 26. The dual space $\mathcal{H}^{\prime}$ is not first countable.

Proof. Assume, by contradiction, that $\mathcal{H}^{\prime}$ is first countable. By Fact $25 \mathcal{H}^{\prime}$ is separable. The following Lemma tells us that under these assumptions $\mathcal{H}^{\prime}$ must be second countable. This lemma, along with its proof, is a standard result that can be found in many treatments of topological vector spaces, including $[5-8,10]$.

Lemma 2. If $X$ is a separable first countable topological vector space, then $X$ is second countable.

Proof. Let $\left\{x_{n}\right\}_{n=1}^{\infty}$ be a countable dense subset of $X$ and let $\left\{B_{k}\right\}_{k=1}^{\infty}$ be a balanced countable local base. We assert that the collection $\left\{x_{n}+B_{k}\right\}_{n, k=1}^{\infty}$ is a countable base for the topology on $X$. To see this let $U$ be an nonempty open set in $X$ with $x \in U$. Since $\left\{B_{k}\right\}_{k=1}^{\infty}$ is a base, there exists an integer $k>0$ such that $x+B_{k} \in U$. Using continuity of addition there exists an integer $k^{\prime}>0$ such that

$$
B_{k^{\prime}}+B_{k^{\prime}} \subset B_{k}
$$

Consider the open set about $x$ given by $x+B_{k^{\prime}}$. Because $\left\{x_{n}\right\}_{n=1}^{\infty}$ is a dense set there exists an integer $n>0$ such that $x_{n} \in x+B_{k^{\prime}}$.

We first show that $x \in x_{n}+B_{k^{\prime}}$. Since $x_{n} \in x+B_{k^{\prime}}$ we have $x_{n}-x \in B_{k^{\prime}}$. Since $B_{k^{\prime}}$ is balanced we must also have $x-x_{n} \in B_{k^{\prime}}$. Thus $x \in x_{n}+B_{k^{\prime}}$.

Now we demonstrate that $x_{n}+B_{k^{\prime}} \subset x+B_{k} \subset U$. Take $z=x_{n}+v$ where $v \in B_{k^{\prime}}$. Then write $z$ as

$$
z=x_{n}+\left(x-x_{n}\right)+v
$$

Noting that $x-x_{n} \in B_{k^{\prime}}$ and $v \in B_{k^{\prime}}$ gives us that $z \in x_{n}+B_{k^{\prime}}+B_{k^{\prime}} \subset x_{n}+B_{k}$ using (22).

Under the assumption that $\mathcal{H}^{\prime}$ is first countable, we have established that $\mathcal{H}^{\prime}$ is second countable. Also, we recall that $\mathcal{H}^{\prime}$ is a Hausdorff topological vector space and thus regular in the strong or weak topology. Therefore Urysohn's Metrization theorem [17] tells us $\mathcal{H}^{\prime}$ is metrizable. This contradicts Fact 17.

Recall that in Fact 24 we saw the dual space is a sequential space. Thus, $\mathcal{H}^{\prime}$ is a (somewhat rare) example of a topological space that is sequential but not first countable.

\section{Continuous Functions}

Linear functionals automatically stand a better chance of having continuity properties in locally convex spaces because they map convex sets to convex sets. In this section we look at some examples of nonlinear functions.

Fact 27. If $X$ is an infinite-dimensional topological vector space (such as $\mathcal{H}^{\prime}$ ) then the only continuous function on $X$ having compact support is 0 . 
Proof. This is because any compact set in $X$ has empty interior, since $X$, being infinite-dimensional, is not locally compact.

Fact 28. There is a weakly continuous function $S$ on $\mathcal{H}^{\prime}$ which satisfies $0<S \leq 1$, and $S$ equals 1 exactly on $D_{-1}(R)$.

Proof. Let

$$
s_{N}=\hat{e}_{1}^{2}+\cdots+\hat{e}_{N}^{2}
$$

which is clearly weakly continuous. Then

$$
\min \left\{1, e^{R^{2}-s_{N}}\right\}
$$

is also weakly continuous, lies in $(0,1]$, and is equal to 1 if and only if $s_{N} \geq R^{2}$. Then each finite sum

$$
\sum_{N=1}^{m} \frac{\min \left\{1, e^{R^{2}-s_{N}}\right\}}{2^{N}}
$$

is weakly continuous, has values in $(0,1]$, and has maximum possible value $1-2^{-m}$ exactly when $s_{m}$ is $\leq R^{2}$. Hence the uniform limit

$$
S=\sum_{N=1}^{\infty} \frac{\min \left\{1, e^{R^{2}-s_{N}}\right\}}{2^{N}}
$$

is a weakly continuous function, with values in $(0,1]$, and is equal to 1 if and only if all $s_{N}$ are $\leq R^{2}$, i.e., on $D_{-1}(R)$.

For the following recall Fact 11. We assume that there are vectors $e_{1}, e_{2}, \ldots \in \mathcal{H}$ which form an orthonormal basis of $H_{0}$ and, moreover, $\lambda_{n}^{-p} e_{n}$ form an orthonormal basis of $H_{p}$, for all $p \in\{0,1,2, \ldots\}$, where

$$
\lambda_{n}=\left\|e_{n}\right\|_{1} .
$$

We require monotonicity and a Hilbert-Schmidt condition

$$
1 \leq \lambda_{1}<\lambda_{2}<\cdots \quad \text { and } \quad \sum_{n=1}^{\infty} \lambda_{n}^{-2}<\infty .
$$

Fact 29. For the cube $C$ in Fact 11, with assumptions as stated in Fact 11, the function $f$ given on $\mathcal{H}^{\prime}$ by

$$
f(x)= \begin{cases}\inf _{n \geq 1}\left\{\lambda_{n}^{n}-\left|\left\langle x, e_{n}\right\rangle\right|\right\} & \text { for } x \in C \\ 0 & \text { for } x \notin C\end{cases}
$$

is continuous but not weakly continuous.

Proof. Suppose $\left\{x^{(n)}\right\} \subset \mathcal{H}^{\prime}$ and $x^{(n)} \rightarrow x$ where $x$ is on the 'boundary' of the cube, i.e., $\left|\left\langle x, e_{k_{0}}\right\rangle\right|=\lambda_{k_{0}}^{k_{0}}$ for some $k_{0}$. Since $x^{(n)} \rightarrow x$ in $\mathcal{H}^{\prime}$ we have $x^{(n)} \rightarrow x$ in $H_{-p}$ for some $p \in\{0,1,2, \ldots\}$. Hence $\left|\left\langle x^{(n)}, e_{k}\right\rangle\right| \rightarrow$ $\left|\left\langle x, e_{k}\right\rangle\right|=\lambda_{k}^{k}$ 
Now

$$
\begin{aligned}
\left|f\left(x^{(n)}\right)-f(x)\right| & =\left|f\left(x^{(n)}\right)-0\right| \\
& =\inf _{k} \lambda_{k}^{k}-\left|\left\langle x^{(n)}, e_{k}\right\rangle\right| \\
& \leq \lambda_{k_{0}}^{k_{0}}-\left|\left\langle x^{(n)}, e_{k_{0}}\right\rangle\right|, \text { for the } k_{0} \text { mentioned above, } \\
& =\lambda_{k_{0}}^{k_{0}}-\left|\left\langle x^{(n)}, e_{k_{0}}\right\rangle\right|
\end{aligned}
$$

and the last term goes to 0 as $n \rightarrow \infty$.

Now consider $x^{(n)} \rightarrow x \notin C$ in $\mathcal{H}^{\prime}$. Then for some $N$ we have for all $n>N, x^{(n)}$ is outside the cube. Thus $f\left(x^{(n)}\right)=0$ for all $n>N$ and hence $f\left(x^{(n)}\right) \rightarrow f(x)=0$ as $n \rightarrow \infty$.

Finally, consider $x^{(n)} \rightarrow x \in C$ in $\mathcal{H}^{\prime}$. Then $x^{(n)} \rightarrow x$ in $H_{-p}$ for some $p$. In particular,

$$
M=\sup _{n}\left|x^{(n)}\right|_{-p}^{2}<\infty
$$

Lemma 3. There exists an $N>0$ such that for any $y \in H_{-p}$ with $|y|_{-p}^{2} \leq M$ we have

$$
\inf _{k \geq 1}\left\{\lambda_{k}^{k}-\left\langle y, e_{k}\right\rangle\right\}=\min \left\{\lambda_{1}-\left|\left\langle y, e_{1}\right\rangle\right|, \lambda_{2}^{2}-\left|\left\langle y, e_{2}\right\rangle\right|, \ldots, \lambda_{N}^{N}-\left|\left\langle y, e_{N}\right\rangle\right|\right\} .
$$

Proof. Since $|y|_{-p}^{2} \leq M$ we have,

$$
|y|_{-p}^{2}=\sum_{k} \lambda_{k}^{-2 p}\left|\left\langle y, e_{k}\right\rangle\right|^{2} \leq M
$$

and consequently

$$
\left|\left\langle y, e_{k}\right\rangle\right| \leq \lambda_{k}^{p} M \quad \text { for all } k \in\{1,2,3, \ldots\}
$$

Therefore

$$
\lambda_{k}^{k}-\left|\left\langle y, e_{k}\right\rangle\right| \geq \lambda_{k}^{k}-\lambda_{k}^{p} M
$$

Since $p$ and $M$ are fixed, there must be an integer $N$ such that for all $k \geq N$

$$
\lambda_{k}^{k}-\left|\left\langle y, e_{k}\right\rangle\right| \geq \lambda_{k}^{k}-\lambda_{k}^{p} M \geq \lambda_{1}
$$

because

$$
\lambda_{k}^{k}-\lambda_{k}^{p} M=\lambda_{k}^{p}\left(\lambda_{k}^{k-p}-M\right) \rightarrow \infty \quad \text { as } k \rightarrow \infty .
$$

Hence, the infimum is realized in the first $N$ terms:

$$
\inf _{k \geq 1}\left\{\lambda_{k}^{k}-\left|\left\langle y, e_{k}\right\rangle\right|\right\}=\min \left\{\lambda_{1}-\left|\left\langle y, e_{1}\right\rangle\right|, \lambda_{2}^{2}-\left|\left\langle y, e_{2}\right\rangle\right|, \ldots, \lambda_{N}^{N}-\left|\left\langle y, e_{N}\right\rangle\right|\right\}
$$

Returning to the proof for Fact 28, by Lemma 3 to $x^{(n)} \rightarrow x$ we have

$$
\begin{aligned}
\lim _{n \rightarrow \infty} f\left(x^{(n)}\right) & =\lim _{n \rightarrow \infty} \min \left\{\lambda_{1}-\left|\left\langle x^{(n)}, e_{1}\right\rangle\right|, \lambda_{2}^{2}-\left|\left\langle x^{(n)}, e_{2}\right\rangle\right|, \ldots, \lambda_{N}^{N}-\left|\left\langle x^{(n)}, e_{N}\right\rangle\right|\right\} \\
& =\min \left\{\lambda_{1}-\left|\left\langle x, e_{1}\right\rangle\right|, \lambda_{2}^{2}-\left|\left\langle x, e_{2}\right\rangle\right|, \ldots, \lambda_{N}^{N}-\left|\left\langle x, e_{N}\right\rangle\right|\right\} \\
& =f(x) \quad \text { (by the Lemma). }
\end{aligned}
$$

Thus, $f$ is sequentially continuous and hence continuous. 


\section{Schwartz Space}

The paper [18] examines the Schwartz space of test functions and the dual space of distributions. There are, of course, many other references for the Schwartz space, including, but not limited to $[15,19-21]$. The Schwartz space is perhaps the best example of a nuclear space to which all the results of this article apply. In particular, the Schwartz space satisfies the structure introduced in Fact 11. In this section we briefly provide an overview of the Schwartz space from [18].

We denote the Schwartz space by $S(\mathbb{R})$ and define it as the space of real-valued infinitely differentiable rapidly decreasing functions on $\mathbb{R}$. However, here we outline how to reconstruct the Schwartz space as a nuclear space arising from $L^{2}(\mathbb{R})$ and the number operator $N=-\frac{d^{2}}{d x^{2}}+\frac{x^{2}}{4}-\frac{1}{2}$.

In [18] we see that orthonormal basis for $L^{2}(\mathbb{R})$ is formed by the functions

$$
\phi_{n}(x)=(-1)^{n} \frac{1}{\sqrt{n !}}(2 \pi)^{-1 / 4} e^{x^{2} / 4} \frac{d^{n} e^{-x^{2} / 2}}{d x^{n}} \quad \text { for } n=0,1,2, \ldots
$$

which are eigenfunctions of $N$. In particular,

$$
N \phi_{n}=n \phi_{n} \quad \text { for } n=0,1,2, \ldots
$$

Using this orthonormal basis and the operator $N$, an inner-product can be defined for any $p \geq 0$

$$
\langle f, g\rangle_{p}=\left\langle(N+1)^{p} f,(N+1)^{p} g\right\rangle_{L^{2}}=\sum_{n=0}^{\infty}(n+1)^{2 p}\left\langle f, \phi_{n}\right\rangle_{L^{2}}\left\langle g, \phi_{n}\right\rangle_{L^{2}}
$$

for $f, g \in S(\mathbb{R})$. (Note: $N+1$ is a Hilbert-Schmidt operator.) Using $\|\cdot\|_{p}$ to denote the norm corresponding to the above inner product we complete $S(\mathbb{R})$ with respect to these norms to form the spaces $S_{p}(\mathbb{R})=\left\{f \in L^{2}(\mathbb{R}) ;\|f\|_{p}<\infty\right\}$. These observations give us

$$
\|\cdot\|_{L^{2}}=\|\cdot\|_{0} \leq\|\cdot\|_{1} \leq \cdots
$$

and

$$
S(\mathbb{R})=\bigcap_{p=0}^{\infty} S_{p}(\mathbb{R}) \subset \cdots \subset S_{2}(\mathbb{R}) \subset S_{1}(\mathbb{R}) \subset L^{2}(\mathbb{R})
$$

just as in (8) and (9).

Also, based on the equation in (27) and (26), $\left\{\phi_{n}\right\}_{n=0}^{\infty}$ forms an orthogonal basis for each $S_{p}(\mathbb{R})$. In particular the structure introduced in Fact 11 is satisfied with $\lambda_{n}=n+1$. Hence all of the results of this article can be applied to the Schwartz space and its dual space.

\section{Closing Remarks}

We have presented an overview of the theory of nuclear spaces in a form that is close to the needs of applications. This included new results along with some previously unknown results and examples. The interested reader can refer to [16] for more on this subject in the same vein, including a discussion of $\sigma$-algebras formed on these spaces.

In Section 8 we explored one application of nuclear spaces. The interested reader is encouraged to explore other related areas, including white noise distribution theory [22-24], Brownian motion [25], Kondratiev's spaces of stochastic distribution [26], and their use in stochastic processes and stochastic linear systems [27,28], among others.

Acknowledgments: A first version of this paper was written when Becnel was supported by NSA grant H98230-10-1-0182. Sengupta's research is supported by NSA grants H98230-13-1-0210 and H98230-15-1-0254. Also, the authors are very grateful to the three referees for their remarks and comments.

Author Contributions: This work is a collaboration between the authors Jeremy Becnel and Ambar Sengupta.

Conflicts of Interest: The authors declare no conflict of interest. 


\section{References}

1. Glimm, J.; Jaffe, A. Quantum Physics: A Functional Integral Point of View; Springer: New York, NY, USA, 1987.

2. Rivasseau, V. From Peturbative to Constructive Renormalization; Princeton University Press: Princeton, NJ, USA, 1991.

3. Itô, K. Foundations of Stochastic Differential Equations in Infinite Dimensional Spaces; Society for Industrial and Applied Mathematics: Philadelphia, PA, USA, 1948.

4. Chiang, T.S.; Kallianpur, G.; Sundar, P. Propagation of Chaos and the McKean-Vlasov Equation in Duals of Nuclear Spaces. Appl. Math. Optim. 1991, 24, 55-83.

5. Grothendieck, A. Topological Vector Spaces (Notes on Mathematics and Its Applicatons); Gordon and Breach: New York, NY, USA, 1973.

6. Robertson, A.; Robertson, W. Topological Vector Spaces; Cambridge University Press: London, UK, 1964.

7. Schäfer, H.H. Topological Vector Spaces; The Macmillan Company: New York, NY, USA, 1966.

8. Köthe, G. Topological Vector Spaces I; Springer: Berlin, Germany, 1969; Volume 1.

9. Wilansky, A. Modern Methods in Topological Vector Spaces; McGraw-Hill: New York, NY, USA, 1978.

10. Pietsch, A. Nuclear Locally Convex Spaces; Springer: Berlin/Heidelberg, Germany; New York, NY, USA, 1965.

11. Rudin, W. Functional Analysis, 2nd ed.; McGraw-Hill: New York, NY, USA, 1991.

12. Driver, B. Analysis Tools with Applications; Springer: Berlin, Germany, 2003.

13. Conway, J.B. A Course in Operator Theory; Springer: Berlin, Germany, 2000.

14. Murphy, G.J. C*-Algebras and Operator Theory; Harcourt Brace Jovanovich: Boston, MA, USA, 1990.

15. Gel'fand, I.M.; Vilenkin, N.Y. Generalized Functions: Application of Harmonic Analysis; Academic Press: New York, NY, USA, 1964; Volume 4.

16. Becnel, J.J. Equivalence of Topologies and Borel Fields for Countably-Hilbert Spaces. Proc. AMS 2006, 134, 581-590.

17. Munkres, J.R. Topology, 2nd ed.; Prentice Hall Inc.: Upper Saddle River, NJ, USA, 2000.

18. Becnel, J.J.; Sengupta, A.N. The Schwartz Space: Tools for Quantum Theory and Infinite Dimensional Analysis. Mathematics 2015, 3, 527-562.

19. Gel'fand, I.M.; Vilenkin, N.Y. Generalized Functions: Spaces of Fundamental and Generalized Functions; Academic Press: New York, NY, USA, 1968; Volume 2.

20. Rudin, W. Functional Analysis; McGraw-Hill: New York, NY, USA, 1987.

21. Simon, B. Distributions and Their Hermite Expansions. J. Math. Phys. 1971, 12, 140-148.

22. Kuo, H.H. White Noise Distribution Theory; Probability and Stochastic Series; CRC Press Inc.: New York, NY, USA, 1996.

23. Obata, N. White Noise Calculus and Fock Space; Lecture Notes in Mathematics; Springer: New York, NY, USA, 1994.

24. Hida, T.; Kuo, H.H.; Potthoff, J.; Streit, L. White Noise: An Infinite Dimensional Calculus; Kluwer Academic Publishers: Norwell, MA, USA, 1993.

25. Hida, T. Analysis of Brownian Functional; Carleton Mathematical Lecture Notes; Carleton University: Ottawa, ON, Canada, 1975.

26. Berezanskiǔ, I.; Kondratiev, I. Spectral Methods in Infinite-Dimensional Analysis; Mathematical Physics and Applied Mathematics; Kluwer Academic: Boston, MA, USA, 1995.

27. Alpay, D.; Salomon, G. Non-commutative stochastic distributions and applications to linear systems theory. Stoch. Process. Appl. 2013, 123, 2303-2322.

28. Alpay, D.; Levanony, D. Linear Stochastic Systems: A White Noise Approach. Acta Appl. Math. 2010, 110, 545-572.

(C) 2016 by the authors; licensee MDPI, Basel, Switzerland. This article is an open access article distributed under the terms and conditions of the Creative Commons Attribution (CC-BY) license (http://creativecommons.org/licenses/by/4.0/). 